\title{
Frequency response of Diamond-like Nanocomposite thin film based MIM capacitor and equivalent circuit modelling
}

\author{
Sukhendu Jana ${ }^{\mathrm{a}}$, Sayan Das ${ }^{\mathrm{a}}$, Utpal Gangopadhyay ${ }^{\mathrm{a}}$, Prajit Ghosh ${ }^{\mathrm{a}}$ and Anup \\ Mondal $^{\mathrm{b}}$ \\ ${ }^{a}$ Meghnad Saha Institute of Technology, Techno India Group, Kolkata - 150, India \\ ${ }^{b}$ Dept. of Chemistry, Bengal Engineering and Science University, Howrah-03, India
}

\begin{abstract}
The frequency response of metal-insulator-metal (MIM) based thin film capacitors were studied using LCR bridge where diamond-like nanocomposite (DLN) film behaves as a dielectric medium. The films were deposited by plasma assisted chemical vapour deposition (PACVD) method. Fourier transforms infrared spectroscopy (FTIR) and Raman spectroscopy give the structure of DLN film. The results show that, equivalent parallel capacitance $(E P C)$ decreases sharply beyond $10^{5} \mathrm{~Hz}$ for thinner films. But for thicker films, there is no such decrease. This is due to some parasitic series resistance effect in the capacitor circuit. An equivalent circuit model for real capacitor has been established. Moreover, there is also a small decrement in EPC with frequency and this effect increases with thickness of film. This may be due to lack of sufficient time for electron transportation through bulk DLN material. The DLN based thin film capacitor has a great potential for use in electronic/electrical system.
\end{abstract}

Keywords: DLN, PACVD, frequency response capacitance, thin film capacitor, etc.

\section{Introduction}

Diamond-like Nanocomposite, an amorphous material comprises of two interpenetrating network structures. These are one diamond like carbon bonds network stabilized by hydrogen $(\mathrm{a}-\mathrm{C}: \mathrm{H})$ and another quartz like silicon network stabilized by oxygen (a-Si:O) [1-4].Different chemical bonds between constituent atoms of the two networks lead to low residual stress[8]. Apart from different tribological $[3,4,6]$, thermal $[4,5]$, chemical [10], biocompatibility [7] properties, DLN has also good dielectric properties [1, 2]. DLN possess electrical resistivity of $10^{8}$ to $10^{14} \mathrm{ohm} \mathrm{cm}$, dielectric constant 3 to 10 and breakdown strength in the range of $10^{6}$ to $10^{8} \mathrm{~V} / \mathrm{cm}[1,2,9]$. Capacitor has been fabricated with DLN thin film as the dielectric medium sandwiched by two metal electrodes. Measurement of frequency response of capacitor fabricated by us in the frequency range of $10^{2} \mathrm{~Hz}$ to $10^{6} \mathrm{~Hz}$ has been reported. An equivalent circuit that represents the real behaviour of the capacitor in electric circuit has also been modelled.

\subsection{Synthesis of DLN film:}

\section{Experimental}

Diamond-like Nanocomposite coatings were deposited on aluminium coated silicon substrate by plasma assisted chemical vapour deposition (PACVD) process. The base pressure is $2 \times 10^{-5} \mathrm{mbar}$ and working pressure is $3 \times 10^{-4}$ mbar. Substrates are cleaned by conventional method and again cleaned in situ by plasma etching prior to deposition. A liquid siloxane precursor injected in the low pressure chamber vapourises with a hot filament and in turn ionizes by bombarding thermionic electrons from filament to generate the plasma atmosphere of precursor elements. The filament current is in the range of 90-110A. The ions are then pulled towards a rotating substrate holder by using a high frequency RF self-bias of $-550 \mathrm{~V}$. During deposition process, argon gas flows in to the chamber at the rate of $50 \mathrm{ml} / \mathrm{min}$.

\section{2: Fabrication of DLN based capacitor:}

Silicon is cleaned by conventional cleaning method and the aluminium thin film is deposited on silicon substrate by vacuum evaporation (PVD) method. Then the DLN film is deposited on aluminium coated silicon substrate by PACVD method. Finally, the aluminum thin film is again deposited on DLN surface. The contact points are achieved by using silver pest. A typical structure of the capacitor is shown in Fig. 1. 


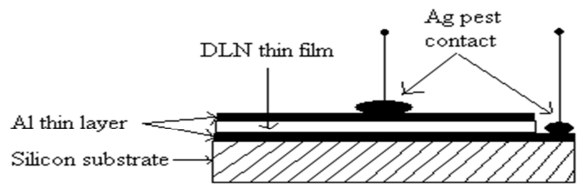

Figure.1. Schematic diagram of single layer DLN based thin film capacitor

\section{Results and discussion}

In order to study of structure of DLN film, the FTIR spectrum of a representative film was recorded from $400-4000 \mathrm{~cm}^{-1}$ range. The corresponding FTIR trace has been shown in Fig.2. The FTIR trace of the film shows two networks: one is Diamond-Like C:H network and another is Si:O network and they are interpenetrated with the Si-C bonding depicting the typical nature of DLN film [3].

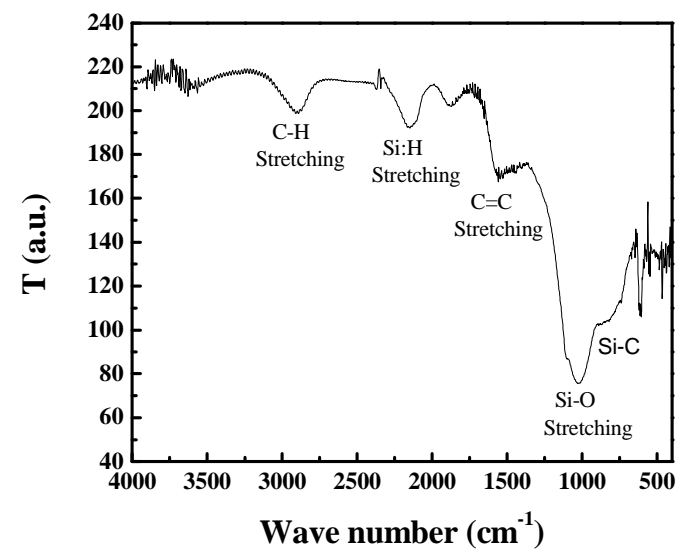

Figure. 2. The FTIR spectra of DLN film shows the bonding structure.

Raman spectrum was investigated in the wave number ranging from 1100 to $1800 \mathrm{~cm}^{-1}$. Raman spectrum analysis of DLN film is deconvoluted into two Gaussian peaks. The G-peak is due to $\mathrm{C}=\mathrm{C} \mathrm{sp}^{2}$ stretching vibration and the D-peak is attributed to the disordered breathing motion of six-fold aromatic rings [3,5]. Thus, the peak positions in the Raman spectrum of DLN film and the intensity ratio of D/G-peak are the most important parameters to understand the electrical properties of the DLN film because $\mathrm{sp}^{2} \pi$ bonds are responsible for electron transportation through DLN film. There are two bands around $1355 \mathrm{~cm}^{-1}$ (D-peak) and $1524 \mathrm{~cm}^{-1}$ (Gpeak) shown in Fig.3. The $\mathrm{I}_{\mathrm{D}} / \mathrm{I}_{\mathrm{G}}$ ratio is observed to be 0.35 which may be indirectly related with $\mathrm{sp}^{3} / \mathrm{sp}^{2}$ ratio [11].

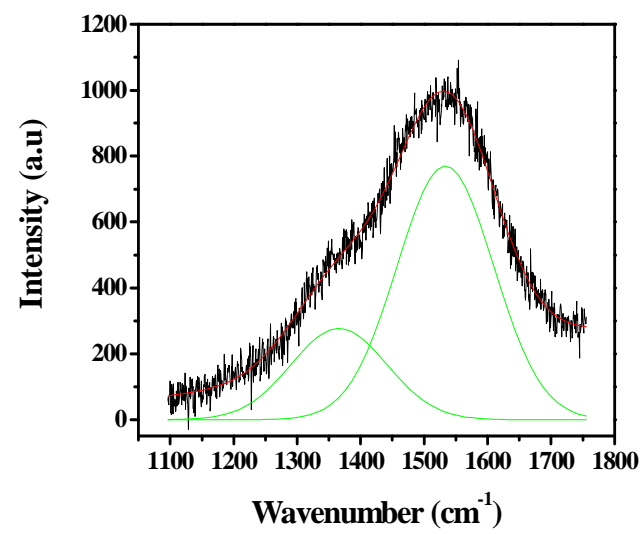

Figure.3. Raman spectra of DLN film is deconvulated into two Gaussian peaks. 


\subsection{Frequency response of EPC:}

The frequency response of EPC of DLN based thin film MIM capacitor has been recorded using Agilent 4284A Precision LCR Meter at room temperature. The peak to peak voltage of ac signal was $0.5 \mathrm{~V}$ with frequency ranges from $10^{2} \mathrm{~Hz}$ to $10^{6} \mathrm{~Hz}$. The Frequency response of three single layer DLN thin films based MIM capacitors has been shown in Fig. 5 and Fig.6. The thickness of DLN films are 118nm, 313nm and $1090 \mathrm{~nm}$ respectively. It is observed that the EPC is almost constant up to $10^{5} \mathrm{~Hz}$ of ac source then decreases sharply for both of thin capacitors. This may be due to some parasitic series resistance effect. We applied an equivalent model for real capacitor to explain such behaviour in section 3.2. Moreover, a small gradient of EPC with frequency is present in all capacitors and the rate of such decrement increases with thickness of film. This may be due to lack of sufficient time to transport of charge through bulk DLN film.

\subsection{Equivalent capacitor model:}

In real capacitor, there must be a definite leakage resistance in between two electrodes and a small series resistance due to resistance of thin metal electrodes and contact terminals. These resistances are termed as parasitic resistance. The LCR bridgemeasured the impedance by assuming a parallel combination of capacitor and resistance i.e. equivalent parallel capacitance (Cp) and equivalent parallel resistance (Rp).
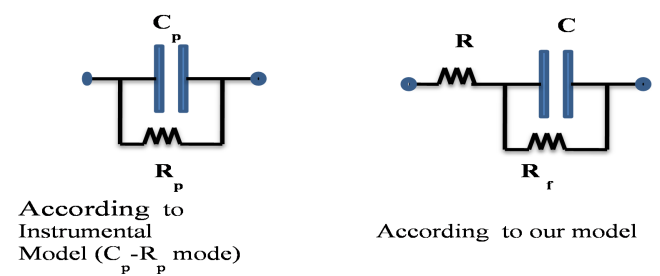

According to our model

Figure 4: Diagram of instrumental model circuit and equivalent model circuit as considered.

According to the instrumental set up, total impedance measured by LCR bridge is converted in two impedances: one is due to equivalent parallel capacitance $\left(C_{p}\right)$ and another is due to equivalent parallel resistance $\left(R_{p}\right)$. According to our model (Fig. 4), we assumed that an ideal capacitor (C) which is independent of frequency $(\sim \mathrm{MHz})$, a parallel leakage resistance $\left(\mathrm{R}_{\mathrm{f}}\right)$ and parasitic resistance $\left(\mathrm{R}_{\mathrm{s}}\right)$ in series.

Total impedance $\left(\mathrm{Z}_{\mathrm{ex}}\right)$ according to instrumental set up is divided as a real part and imaginary part. Similarly, total impedance of equivalent model circuit is divided in real and imaginary parts. Comparing the real and imaginary part of equivalent model circuit and instrumental circuit, we get,

$$
\begin{aligned}
& \mathbf{C}_{\mathbf{p}}=\frac{\mathbf{C R}_{\mathbf{f}}^{2}}{\left(\mathbf{x R}_{\mathrm{s}}^{2}+2 \mathbf{R}_{\mathbf{s}} \mathbf{R}_{\mathbf{f}}+\mathbf{R}_{\mathrm{f}}^{2}\right)} \ldots \ldots \ldots \ldots \ldots \ldots 1 \\
& \mathbf{R}_{\mathbf{p}}=\frac{\left(\mathbf{x R}_{\mathbf{s}}^{2}+2 \mathbf{R}_{\mathbf{s}} \mathbf{R}_{\mathbf{f}}+\mathbf{R}_{\mathbf{f}}^{2}\right)}{\left(\mathbf{x R}_{\mathbf{s}}+\mathbf{R}_{\mathbf{f}}\right)} \ldots \ldots \ldots \ldots \ldots \ldots 2 \\
& \text { where } \mathrm{x}=1+\omega^{2} \mathrm{C}^{2} \mathbf{R}_{\mathrm{f}}^{2} \ldots \ldots \ldots \ldots \ldots 3
\end{aligned}
$$

In general, the series resistance $\left(R_{s}\right)$ is much smaller than the leakage resistance $\left(R_{\mathrm{f}}\right)$. At low frequency, ' $\mathrm{x}$ 'in equation 1 is closes to one hence; $\mathrm{Cp}$ and $\mathrm{Rp}$ are nearly equal to $\mathrm{C}$ and $\mathrm{R}_{\mathrm{f}}$ respectively according to equations 1 and 2 . If $R_{f}$ is very high $\left(\sim \mathrm{M} \Omega\right.$ or higher), then $R_{s}$ has no significant effect on EPC even at high frequency $\left(10^{6} \mathrm{~Hz}\right)$. But if $R_{\mathrm{f}}$ is low $(\sim \mathrm{k} \Omega)$, then $\mathrm{R}_{\mathrm{s}}$ can play an important role on EPC at higher frequency. By changing the value of $R_{s}$, we fitted the experimental graph from the equivalent model circuit.

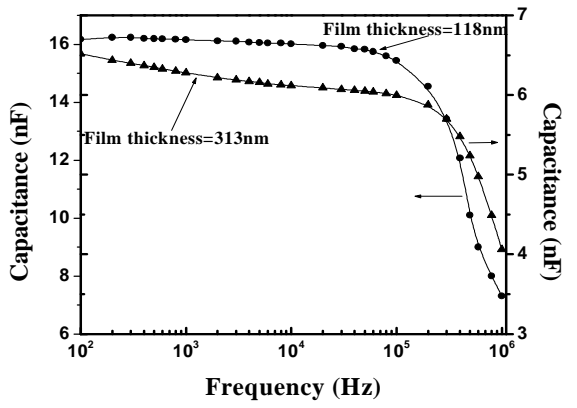

Figure 5: Frequency response of EPC for 118nm and 313nm thick DLN based capacitor. 


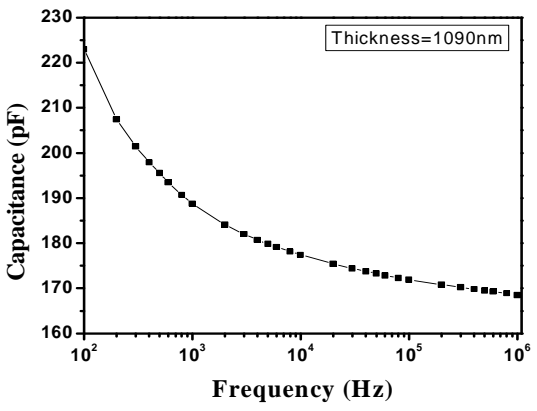

Figure 6: Frequency response of EPC for $1090 \mathrm{~nm}$ thick DLN based capacitor.

The frequency response of EPC of single layer (thickness=118nm) DLN film capacitor has good agreement with equivalent model circuit (Fig. 7). The EPC and EPR of the capacitor are $16.2 \mathrm{nF}$ and $9.48 \mathrm{k} \Omega$ respectively at frequency $100 \mathrm{~Hz}$. So the value of $\mathrm{C}$ and $\mathrm{R}_{\mathrm{f}}$ are $16.2 \mathrm{nF}$ and $9.48 \mathrm{k} \Omega$ respectively.

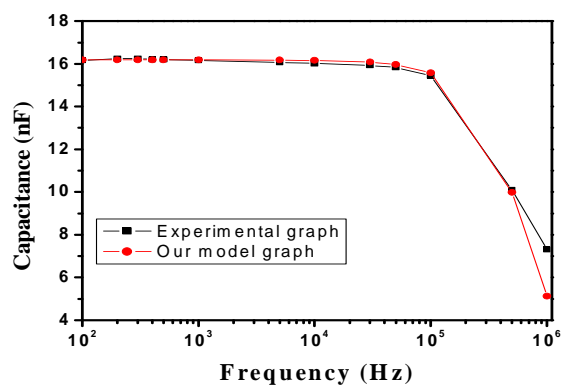

Figure. 7: Capacitance vs. frequency response for DLN single layer thin film capacitor of thickness $118 \mathrm{~nm}$. $\left(\mathrm{R}_{s}\right.$ $=15 \Omega, \mathrm{R}_{\mathrm{f}}=9.48 \mathrm{k} \Omega, \mathrm{C}=16.2 \mathrm{nF}$ )

It has been seen by trial and error method that, $R_{s}$ is equal to $15 \Omega$ for better fitment of experimental results.

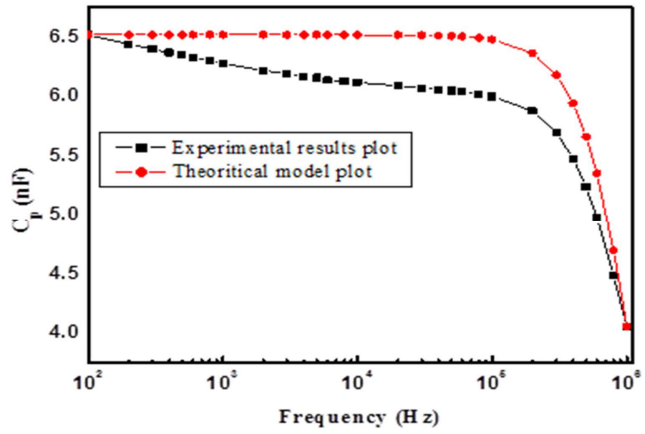

Figure 8: Capacitance vs. frequency response for DLN single layer thin film capacitor of thickness $313 \mathrm{~nm} .\left(\mathrm{R}_{\mathrm{s}}=\right.$ $\left.18 \Omega, \mathrm{R}_{\mathrm{f}}=4.5 \mathrm{M} \Omega, \mathrm{C}=6.52 \mathrm{nF}\right)$.

Fig. 8 also shows that the frequency response of EPC of single layer (thickness=313nm) DLN film capacitor has agreement with theoretical model. The EPC and EPR of the capacitor are $6.52 \mathrm{nF}$ and $4.5 \mathrm{M} \Omega$ respectively at frequency $100 \mathrm{~Hz}$. Similarly, the value of $\mathrm{C}$ and $\mathrm{R}_{\mathrm{f}}$ are $6.52 \mathrm{nF}$ and $4.5 \mathrm{M} \Omega$ respectively. It also has been seen by trial and error method that, $R_{\mathrm{s}}$ is equal to $18 \Omega$ for better fitment of experimental results.

There is no sharp decrement of EPC at high frequency for thick layer DLN film $(1090 \mathrm{~nm})$ which shown in Fig. 6 . Because the leakage resistant is very high comparable to series resistance and hence does not have any effect within frequency $1 \mathrm{MHz}$ on EPC according to equation 1. The EPC and EPR are 223pF and 30M $\Omega$ respectively at $100 \mathrm{~Hz}$ frequency.

Another interesting observation is that, there is a gradual decrease of EPC with frequency up to $10^{5} \mathrm{~Hz}$. This effect increases with film thickness. These may be explained as follows: for any definite potential difference across the capacitor the field strength becomes very high for very thin dielectric film. Due to the high field strength and defect states present in the bulk of the film, transportation of electrons through DLN film may be explained by Pool-Frankle model for insulators. The transportation causes electron drift velocity, so electrons 
take some time to cross the bulk material if applied field remains active in that direction. For alternating electric field, as applied field frequency increases frequent change in field direction causes decrease in transportation of electrons because of lesser available time for them to be transported. Hence current decreases in the circuit i.e. circuit impedance increases. Increase of impedance signifies decrease of EPC. Apart from this when thickness of DLN film increases the field strength decreases for specific potential difference and the path length for electrons also increase as a result time for transportation further increase causing lowering of EPC. When the frequency is quite high $>10^{4} \mathrm{~Hz}$ the decrement in EPC reduces causing more or less a steady capacitance for thicker film (Fig. 9).

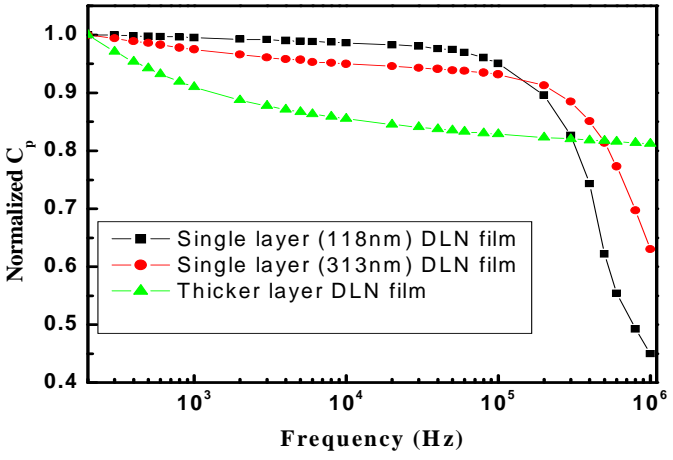

Figure 9. Normalized EPC for four different samples

The sharp decrease in EPC after $10^{5} \mathrm{~Hz}$ for thinner DLN film layered capacitors in Fig 9 may be attributed to parasitic series resistance effect in the capacitor circuit as mentioned in section 3.2.

\section{Conclusion}

The study for capacitance against frequency of DLN thin film based MIM capacitor shows that, parasitic resistance has a great effect on EPC at high frequency operation. To avoid this effect, the leakage resistance should be very high and parasitic resistance should be very low. For a particular dielectric medium to increase the leakage resistance the thickness of dielectric must be enlarged which leads to decrease in capacitance for fixed electrode area. In case of high capacitance and high frequency operation, our model can be helpful to design an electrical/ electronic circuit properly since it has good matching with experimental studies. Hence, DLNthin film based MIM capacitor has great potential for use in electrical/ electronic circuit.

\section{Acknowledgement:}

The authors gratefully acknowledge the DST, Govt. of India for financial support. The FTIR and Raman Spectra studies were carried out in the IIT, Kharagpur, IACS, Kolkata respectively.

\section{Reference}

1. ArvindGoel, Chandra Venkatramanl, Benjamin F Dorfman, Michael Abraizovz, Thomas $\boldsymbol{G}$. Enge1 and Nicholas $\boldsymbol{G}$ Loter, "DIAMOND-LIKE NANOCOMPOSITE COATINGS POSSESSING HIGH DIELECTRIC STRENGTH" 1995 IEEE 5th International Conference on Conduction and Breakdown in Solid Dielectrics, 690.

2. C. Venkatraman, A. Goel, R. Lei, D. Kester, C. Outten, "Electrical properties of diamond-like nanocomposite coatings", Thin Solid Films, 308-309, 1997, 173

3. Won Jae Yanga,, Yong-Ho Choa, TohruSekino, Kwang Bo Shim, Koichi Niihara, Keun Ho Auh, "Structural characteristics of diamond-like nanocomposite films grown by PECVD”, Materials Letters, 57, 2003, 3305.

4. C. Venkatraman , C. Brodbeck, R. Lei, "Tribological properties of diamond-like nanocomposite coatings at high temperatures", Surface and Coatings Technology, 115, 1999, 215.

5. Won Jae Yang, Yong-Ho Choa, TohruSekino, Kwang Bo Shim, Koichi Niihara, Keun Ho Auh, "Thermal stability evaluation of diamond-like nanocomposite coatings", Thin Solid Films, 434, 2003, 49.

6. D. Neerinck, P. Persoone, M. Sercu, A. Goel, D. Kester, D. Bray, "Diamond-like nanocomposite coatings (a-C:H/a-Si:O) for tribological applications", Diamond and Related Materials, 7, 1998, 468.

7. T. Das - D. Ghosh- T. K. Bhattacharyya - T. K. Maiti, "Biocompatibility of diamond-like nanocomposite thin films", J Mater Sci: Mater Med, 18, 2007, 493.

8. A. PANDIT, N. P. PADTURE, "Interfacial toughness of diamond-like nanocomposite (DLN) thin films on silicon nitride substrates", JOURNAL OF MATERIALS SCIENCE LETTERS,22, 2003, 1261.

9. ArvindGoel, Donald J. Bray, Steven C. Martin, Keith A. Blakely, "CAPACITIVE THIN FILMS USING DIAMOND-LIKE NANOCOMPOSITE MATERIALS”, United States Patent [19], Patent Number: 5,638,251, Date of Patent: Jun. 10, 1997.

10. Veniamin F. Dorfman, ArvindGoel, Donald J. Bray, “ EROSION RESISTANT DIAMOND-LIKE NANOCOMPOSITE COATINGS FOR OPTICAL COMPONENTS", United States Patent [19], Patent Number: 5,718,976, Date of Patent: Feb. 17, 1998.

11. G. Mariotto, C. Vinegoni, L.G. Jacobsohn, F.L. FreireJr, "Raman spectroscopy and scanning electron microscopy investigation of annealed amorphous carbon-germanium films deposited by d.c. magnetron sputtering", Diamond and Related Materials, 8, 1999, 668. 